\title{
Whole body diffusion for metastatic disease assessment in neuroendocrine carcinomas: comparison with OctreoScan ${ }^{\circledR}$ in two cases
}

\author{
Rachel Jorge D Cossetti, Regis Otaviano França Bezerra, Brenda Gumz, Adriana Telles and Frederico P Costa*
}

\begin{abstract}
Neuroendocrine tumor (NET) patients must be adequately staged in order to improve a multidisciplinary approach and optimal management for metastatic disease. Currently available imaging studies include somatostatin receptor scintigraphy, like OctreoScan ${ }^{\circledR}$, computed tomography (CT), scans and magnetic resonance imaging (MRI), which analyze vascular concentration and intravenous contrast enhancement for anatomic tumor localization. However, these techniques require high degree of expertise for interpretation and are limited by their availability, cost, reproducibility, and follow-up imaging comparisons. NETs significantly reduce water diffusion as compared to normal tissue. Diffusion-weighted imaging (DWI) in MRI has an advantageous contrast difference: the tumor is represented with high signal over a black normal surrounding background. The wholebody diffusion (WBD) technique has been suggested to be a useful test for detecting metastasis from various anatomic sites. In this article we report the use of DWI in MRI and WBD in two cases of metastatic pulmonary NET staging in comparison with OctreoScan ${ }^{\circledR}$ in order to illustrate the potential advantage of DWI and WBD in staging NETs.
\end{abstract}

Keywords: Neuroendocrine tumor, Diffusion-weighted image, Magnetic resonance image, OctreoScan ${ }^{\circledR}$

\section{Background}

Neuroendocrine tumors (NETs) are heterogeneous malignant neoplasms that originate from neuroendocrine cells located in various anatomic sites of the body. They are classically associated with symptoms resulting from secretion of hormones or vasoactive peptides into the systemic circulation, and classified as functioning and non-functioning tumors. The Surveillance, Epidemiology, and End Results Program database reported the incidence of 5.25 cases per 100,000 for the 2004 United States population [1]. The long survival experienced by NET patients explains its high prevalence, which was estimated in $35 / 100,000$ in the same population. Approximately $50 \%$ of NET patients are diagnosed with localized disease and surgical resection alone is often curative.

The liver is a frequent site of metastasis, with substantial influence on prognosis [2-5]. Patients who present

\footnotetext{
* Correspondence: fredericoperegocosta@gmail.com

Centro de Oncologia, Hospital Sírio Libanês, Rua Dona Adma Jafet 91, São Paulo 01308-050, Brazil
}

with advanced disease limited to the liver may also benefit from surgical resection with potential curative intent or to control hormone secretion refractory to biotherapy or other systemic treatment options. In order to better select candidates for surgical procedure, clinical staging is fundamental.

Despite great technological improvement in imaging acquisition in recent years, detection of hepatic lesions less than $5 \mathrm{~mm}$ in diameter is still limited. Therefore, diffusion-weighted imaging (DWI) in MRI has recently emerged as a tool for detecting metastasis in the liver without the need of i.v. contrast or radiation exposure [6,7]. DWI sequence can be adjusted to obtain images from the entire body in one single acquisition called whole-body diffusion imaging (WBD). To our knowledge, only limited studies have addressed the value and the practicality of MRI diffusion in staging NETs [8,9]. The objective of this report is to assess the DWI and WBD in two patients with metastatic neuroendocrine carcinoma and demonstrate its potential use in comparison with OctreoScan ${ }^{\circledR}$, commonly used in the clinical staging of NET. 


\section{Case presentation}

We report two patients with metastaic neuroendocrine carcinoma submitted to MRI with DWI and WBD and OctreoScan ${ }^{\circledR}$ in their clinical staging. The imaging protocols and medical history is presented bellow.

\section{MRI image protocol}

MRI was performed with a 1.5-T whole-body imager (Signa HDx; General Electric Medical Systems, Milwaukee, WI, USA). For every scan, whole-body examination was obtained in axial and coronal plane using body coil. Three sequences were obtained and the image parameters are described in the Table 1.

For each sequence, at least five continuous stations covering the whole body from the top of the head to feet were acquired. Estimated examination time is up to $30 \mathrm{~min}$.

The diffusion encoding was done in only one direction and the lesions detected in the diffusion-weighted sequence were analyzed in terms of number, size, location, and signal intensity. These lesions were compared to the T1-weighted and STIR (Short TI Inversion Recovery) sequences to rule out benign lesions (false-positive).

Using quantitative analysis, an apparent diffusion coefficient map (ADC map) was calculated for the main lesions in each patient. After these analyses, all lesions considered malignant were correlated with OctreoScan ${ }^{\circledR}$ with time interval between the exams of less than one week.

\section{Somatostatin receptor scintigraphy}

Somatostatin receptor scintigraphy (SRS) is a functional technique for the imaging of NETs. There is a variety of

\begin{tabular}{|c|c|c|c|}
\hline Parameter & DWI & $\mathrm{T} 1$ & STIR \\
\hline$\overline{\mathrm{TR} / \mathrm{TE}}$ & $6750 / 64$ & $1000 / 4.6$ & $3200 / 60$ \\
\hline Inversion time (ms) & 180 & - & 180 \\
\hline Field of view (mm) & 450 & 450 & 450 \\
\hline Matrix size & $100 \times 128$ & $256 \times 224$ & $256 \times 128$ \\
\hline $\begin{array}{l}\text { Section thickness/ } \\
\text { gap }(\mathrm{mm})\end{array}$ & 5 & 5 & 5 \\
\hline $\begin{array}{l}\text { Parallel acquisition } \\
\text { technique factor }\end{array}$ & - & - & - \\
\hline Signals averaged $(n)$ & 6 & 1 & 2 \\
\hline Blocks $(n)$ & 5 & 5 & 5 \\
\hline Acquisition time & $\begin{array}{l}2 \text { min } 30 \mathrm{~s} \\
\text { for each block }\end{array}$ & $\begin{array}{l}32 \mathrm{~s} \text { for } \\
\text { each block }\end{array}$ & $\begin{array}{l}1 \text { min } 40 \text { s for } \\
\text { each block }\end{array}$ \\
\hline B-value (s/mm2) & 0,600 & - & - \\
\hline Bandwidth $(\mathrm{kHz})$ & 125 & 62.5 & 50 \\
\hline
\end{tabular}

Dash (-) indicates not applicable.

DWI, diffusion-weighted imaging; STIR, Short T1 Inversion Recovery. peptides (known as somatostatin analogues) which target these receptors, and which differ in their ability to bind to the various receptor subtypes (SSTR). The most commonly used somatostatin analogue is octreotide which is labeled with 111In, using the chelator diethylenetriamine-pentaaceticacid (DTPA), to produce 111In-DTPAoctreotide [6-11] which is available as a commercial product (OctreoScan ${ }^{\circledR}$-Mallinckrodt, Inc.). OctreoScan ${ }^{\circledR}$ was supplied as two vials: Vial A - 111In as InCl3, $122 \mathrm{MBq}(3.3 \mathrm{mCi}) / 1.1 \mathrm{ml}$; Vial B - $10 \mu \mathrm{g}$ of lyophilized pentetreotide and excipients. The maximum amount of IV pentetreotide injected is $10 \mu \mathrm{g}$.

OctreoScan ${ }^{\circledR}$ images are obtained by using a gamma camera fitted with a medium-energy, parallel hole collimator (Symbia T2, Siemens) with hybrid technique SPECT/CT (single-photon emission computed tomography and digital computed tomography at the same study). Planar and SPECT images were acquired at 4 and $24 \mathrm{~h}$ post-injection.

\section{Medical history \\ Patient 1}

A 31-year-old female underwent dermatological evaluation for resection of a subcutaneous nodule. Pathological analysis demonstrated an adenocarcinoma with positive margins. The patient underwent wide excision of surgical margins and pathology revealed a NET with positive staining for chromogranin $\mathrm{A}$ and neuronspecific enolase. A chest CT scan showed a pulmonary mass in the left lobar bronchia. The patient underwent left pneumectomy. Pathology confirmed a $4.5-\mathrm{cm}$ welldifferentiated neuroendocrine carcinoma, with a low mitotic count of 1 mitosis per $10 \mathrm{HPF}$ (high power field). There was no vascular or pleural invasion and surgical margins were free. One lymph node was involved. A staging abdominal ultrasound revealed the presence of two hepatic nodules in segment VIII, measuring 1.8 and $1.0 \mathrm{~cm}$. An abdominal MRI showed multiple hepatic nodules, with additional lesions in segments II and V. Octreoscan showed increased activity on pulmonary and hepatic tumoral lesions. Laboratory screening was unremarkable. The patient was treated with somatostatin analogs. Follow-up abdominal MRI revealed multiple new hepatic and bone lesions. In the WBD images, a new small sacral lesion was detected in correlation with a low signal nodule on the T1-weighted images and therefore raised suspicion for metastasis (Figure $1 \mathrm{~A}$ and $1 \mathrm{C}$ ). The Octreoscan $^{\circledR}$ performed in the same week of the WBD/MRI showed focal uptake in this area (Figure 1B) confirming that WBI added valuable information for staging. Everolimus was added to the treatment regimen. Follow-up images revealed stable disease after three months. 

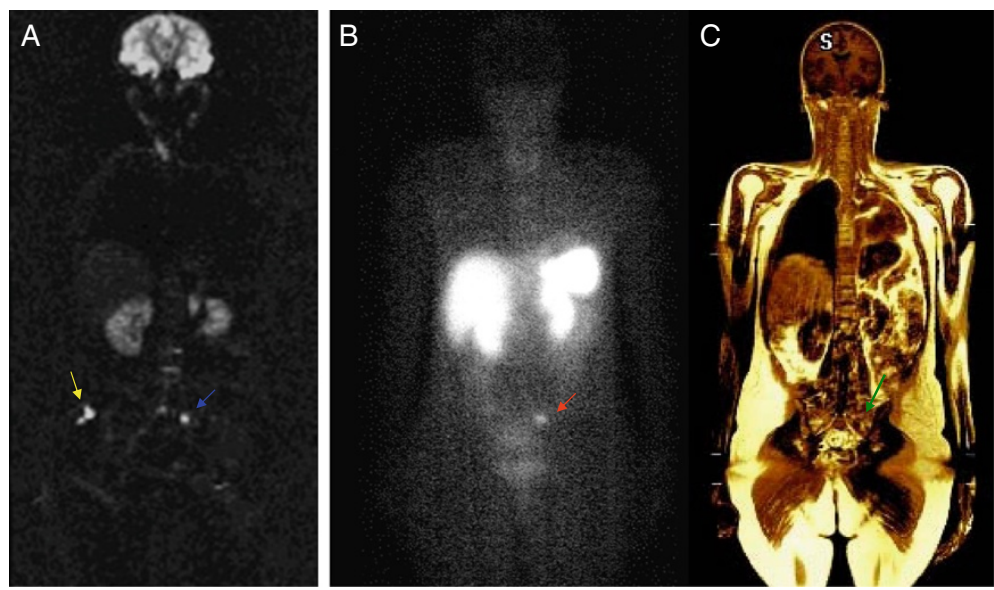

Figure 1 Comparison of DWI (A), 111 In-pentetreotide scintigraphy images (B) and T1-weighted (C) showing bone metastasis in left sacral bone detected by diffusion-weighted imaging (blue arrow) and confirmed by T1 sequence (green arrow). The hyperintense signal in the right subcutaneous fluid (yellow arrow) is a pitfall due to T2 shine-throught effect and should not be interpreted as metastasis. OctreoScan ${ }^{\circledR}$ image (B) reveals correlation between radiotracer uptake (red arrow) and MR findings.

\section{Patient 2}

A 23-year-old male presented with sporadic haemoptysis over the last year. A CT scan of the thorax showed an intra-bronchial lesion with $3.0 \times 2.3 \mathrm{~cm}$ in the right inferior lobar bronchia and a bronchoscopy confirmed a well-differentiated NET, grade 1 . The tumor was a nonfunctioning pulmonary NET-1. He underwent a right inferior lobectomy. Pathology reported an invasive welldifferentiated pulmonary neuroendocrine tumor, grade 1 , measuring $2.2 \mathrm{~cm}$, with predominant endobronchial growth, with less than 1 mitosis per $10 \mathrm{HPF}$. There was neither neural-vascular invasion nor necrosis. According to pathological analysis, zero of two mediastinal lymph nodes were affected (pT1b pN0). Immunohistochemistry revealed a proliferation index (Ki67) of $<1 \%$, with positive staining for chromogranin A, synaptophysin, cytokeratin(CK)-40, CK-48, CK-50,6 and $\mathrm{kDa}$, and negative staining for TTF-1. A staging CT scan of thorax, abdomen, and pelvis demonstrated unexpected small round hypointense lesions distributed throughout the liver parenchyma, seen only on the venous phase, measuring less than $10 \mathrm{~mm}$. Laboratory exams were relevant for serum chromogranin A $38 \mathrm{ng} / \mathrm{mL}$ (normal reference value: < $15 \mathrm{ng} / \mathrm{mL}$ ) and urinary 5-hydroxyindoleacetic acid (5HIAA) $5.7 \mathrm{mg} / 24 \mathrm{~h}$ (normal reference value: $<8.2 \mathrm{mg} /$ $24 \mathrm{~h}$ ). The patient was referred for hepatic resection at our center. An abdominal MRI was performed to evaluate the hepatic lesions, revealing multiple hypervascular nodules scattered throughout the liver with washout on the delayed phase. All these hepatic lesions were detected in the DW images. WBD images revealed, in addition to the hepatic lesions, mediastinal lymph nodes with restricted diffusion confirmed in the ADC maps raising suspicion for metastatic lesions (Figure 2A). The
Octreoscan ${ }^{\circledR}$ performed on the day after showed focal areas of uptake in superior mediastinal, para-tracheal, subcarinal and right pulmonar hilar lymph nodes and hepatic nodules in segments V/VIII and III (Figure 2B). The patient was submitted to a fine needle aspiration biopsy of the large hepatic lesion, which confirmed metastatic neuroendocrine carcinoma. Treatment with somatostatin analogs was initated.

\section{Discussion}

Magnetic resonance or computed tomography (CT) explore NET characteristics of being highly vascular and enhance intensely with intravenous contrast during the early arterial phases with washout in the delayed phases. On the other hand, liver metastasis may appear isodense in pre-contrast and venous phases causing poor visualization. This variation creates challenges in staging and evaluating tumor response or progression during treatment. Multiphase CT, which includes both arterial and portal venous phase images, is often used to stage and access tumor response in patients with NET. Magnetic resonance imaging (MRI) is also a commonly used alternative for imaging NET. Metastatic lesions can be visualized with and without gadolinium contrast, especially in T2-weighted sequences, reducing the variability sometimes seen with CT-based imaging results $[10,11]$. However, both imaging methods require intravenous (i.v.) contrast.

Scintigraphic technique is also commonly used to stage NET by identifying primary and metastatic anatomic sites, especially in well and moderately-differentiated NETs. OctreoScan ${ }^{\circledR}$ specifically binds to SSTR, with particular affinity for subtypes 2, 3, and 5, when they are expressed in tumor. ${ }^{68} \mathrm{Ga}-\mathrm{DOTANOC}$ is 

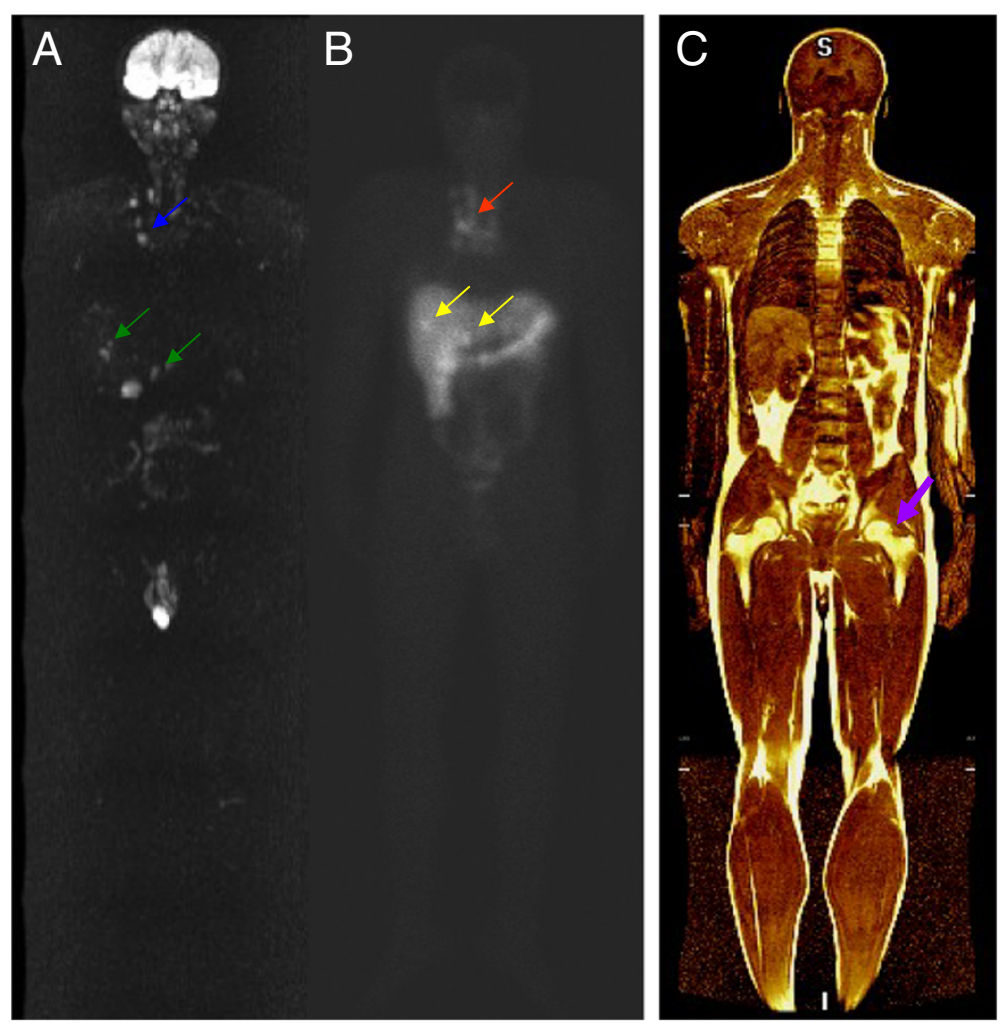

Figure 2 Comparison of DWI (A) and 111 In-pentetreotide scintigraphy (B) images reveal correlation between hyperintense mediastinal lymph nodes (blue arrows) and radiotracer uptake (red arrow). All the metastatic liver lesions detected by scintigraphy (yellow arrows) appeared as hyperintense nodules in the DWI (green arrows). T1-weighted image (C) shows the potential application of this method to detect bone metastasis (purple arrows).

another promising peptide with broader and higher affinity with SSTR. It seems to provide better visualization and relevant information in the management of NET patients compared to OctreoScan ${ }^{\circledR}$, although this new peptide is still not widely available outside a small number of referring centers in the world [12-14].

NETs significantly reduce water diffusion compared to normal tissues. DWI in MRI has an advantageous contrast difference for lesion detection: the tumor is represented with high signal over a black normal surrounding tissue. This is a patient-friendly tool that does not require i.v. contrast, does not use ionizing radiation, and can be correlated with other T1/T2 MRI acquisitions for better tumor location and characterization. The WBD image is a fast acquisition phase and can be easily used as a roadmap to identify small and large NET lesions. Repeated images can also be made in order to assess tumor response, not only with morphological analysis but also providing functional information through quantification of apparent diffusion coefficient (ADC) values. The precise anatomic information, the speed of imaging acquisition, the lack of endovenous contrast, wide availability, and low cost make the whole body diffusion a reliable and attractive staging method that should be further investigated.

WBD has been shown to be the most accurate technique for detecting metastasis of melanoma in the liver, bone, subcutaneous tissue, and intra-peritoneum [15]. WBD has also been used to detect metastatic lesions from paraganglioma and pheochromocytoma, with higher rates of detection than 2-[(18)F]-fluoro-2-deoxyD-glucose positron emission tomography (FDG-PET) or (123)I-meta-iodo-benzyl guanidine scintigraphy (MIBG) [16].

Despite the paucity of diffusion MRI reports in NETs, these two cases illustrate a potential role of DWI and WBD as a cost-effective and less invasive method for assessing NETs. Variations in water diffusion restriction could provide information about tumor response during treatment, with less cost and risks in comparison with other standard methods. We acknowledge that the functional information provided by WBD still needs further evaluation and currently does not substitute OctreoS$\operatorname{can}^{\circledR}$. A formal evaluation in NET patients should be conduct to define the sensitivity and specificity of this method in comparison with other standard imaging 
techniques. DWI and WBD could have a potential and import role for staging NET in surgical candidates and for following up patients on systemic therapy.

\section{Consent}

Written informed consent was obtained from the patients for publication of this case report and any accompanying images. A copy of the written consent is available for review by the Editor-in-Chief of this journal.

\section{Abbreviations}

ADC map: Apparent diffusion coefficient map; CK: Cytokeratin; CT: Computed tomography; DTPA: Diethylenetriamine-pentaaceticacid; DWI: Diffusionweighted image; FDG-PET: 2-[(18)F]-fluoro-2-deoxy-D-glucose positron emission tomography; HPF: High power field; i.v: Intravenous; MIBG: (123)Imeta-iodo-benzyl guanidine scintigraphy; MRI: Magnetic resonance image; NET: Neuroendocrine tumor; SPECT: Single-photon emission computed tomography; SSTR: Somatostatin subtype receptor; STIR: Short TI Inversion Recovery; WBD: Whole-body diffusion; 5-HIAA: 5-hydroxyindoleacetic acid.

\section{Competing interests}

The authors declare that they have no competing interests.

\section{Authors' contributions}

RJC: case preparation and revised the manuscript. The author read and approved the final manuscript; RB: case preparation, established the imaging protocols and revised the manuscript; BG: revised the manuscript; AT: revised the manuscript; FC: case preparation, drafted discussion and revised the manuscript. FC: is member of the advisor board member for the European Neuroendocrine Tumor Society. All the authors read and approved the final manuscript.

Received: 26 August 2011 Accepted: 16 May 2012

Published: 16 May 2012

\section{References}

1. Yao JC, Hassan M, Phan A, Dagohoy C, Leary C, Mares JE, Abdalla EK, Fleming JB, Vauthey J-N, Rashid A, Evans DB: One hundred years after "carcinoid": epidemiology of and prognostic factors for neuroendocrine tumors in 35,825 cases in the United States. J Clin Oncol 2008, 26:30633072.

2. Norton JA: Endocrine tumours of the gastrointestinal tract. Surgical treatment of neuroendocrine metastases. Best Pract Res Clin Gastroenterol 2005, 19:577-583.

3. Tomassetti P, Campana D, Piscitelli L, Casadei R, Santini D, Nori F, MorselliLabate AM, Pezzilli R, Corinaldesi R: Endocrine pancreatic tumors:factors correlated with survival. Ann Oncol 2005, 16:1806-1810.

4. Chamberlain RS, Canes D, Brown KT, Saltz L, Jarnagin W, Fong Y, Blumgart LH: Hepatic neuroendocrine metastases: does intervention alter outcomes? J Am Coll Surg 2000, 190:432-445.

5. McEntee GP, Nagorney DM, Kvols LK, Moertel CG, Grant CS: Cytoreductive hepatic surgery for neuroendocrine tumors. Surgery 1990, 108:1091-1096.

6. Ichikawa T, Haradome H, Hachiya J, Nitatori T, Araki T: Diffusion-weighted MR imaging with single-shot echo-planar imaging in the upper abdomen: preliminary clinical experience in 61 patients. Abdom Imaging 1999, 24:456-461.

7. Gourtsoyianni S, Papanikolaou N, Yarmenitis S, Maris T, Karantanas A, Gourtsoyiannis N: Respiratory gated diffusion-weighted imaging of the liver: value of apparent diffusion coefficient measurements in the differentiation between most commonly encountered benign and malignant focal liver lesions. Eur Radiol 2008, 18:486-492.

8. Liapi E, Geschwind JF, Vossen JA, Buijs M, Georgiades CS, Bluemke DA Kamel IR: Functional MRI evaluation of tumor response in patients with neuroendocrine hepatic metastasis treated with transcatheter arterial chemoembolization. AJR Am J Roentgenol 2008, 190:67-73.
9. Anaye A, Mathieu A, Closset J, Bali MA, Metens T, Matos C: Successful preoperative localization of a small pancreatic insulinoma by diffusionweighted MRI. JOP 2009, 10:528-531.

10. Dromain C, de Baere T, Baudin E, Galline J, Ducreux M, Boige V, Duvillard P, Laplanche A, Caillet H, Lasser P, Schlumberger M, Sigal R: MR imaging of hepatic metastases caused by neuroendocrine tumors: comparing four techniques. AJR Am J Roentgenol 2003, 180:121-128.

11. Debray MP, Geoffroy O, Laissy JP, Lebtahi R, Silbermann-Hoffman O, HenryFeugeas MC, Cadiot G, Mignon M, Schouman-Claeys E: Imaging appearances of metastases from neuroendocrine tumours of the pancreas. Br J Radiol 2001, 74:1065-1070.

12. Ambrosini V, Campana D, Bodei L, Nanni C, Castellucci P, Allegri V, Montini GC, Tomassetti P, Paganelli G, Fanti S: 68 Ga-DOTANOC PET/CT clinical impact in patients with neuroendocrine tumors. J Nucl Med 2010, 51:669673.

13. Campana D, Ambrosini V, Pezzilli R, Fanti S, Labate AM, Santini D, Ceccarelli C, Nori F, Franchi R, Corinaldesi R, Tomassetti P: Standardized uptake values of (68)Ga-DOTANOC PET: a promising prognostic tool in neuroendocrine tumors. J Nucl Med 2010, 51:353-359.

14. Tan EH, Tan CH: Imaging of gastroenteropancreaticneuroendocrine tumors. World J Clin Oncol 2011, 2:28-43.

15. Laurent V, Trausch G, Bruot O, Olivier P, Felblinger J, Régent D: Comparative study of two whole-body imaging techniques in the case of melanoma metastases: advantages of multi-contrast MRI examination including a diffusion-weighted sequence in comparison with PET-CT. Eur J Radiol 2010, 75:376-383.

16. Takano A, Oriuchi N, Tsushima Y, Taketomi-Takahashi A, Nakajima T, Arisaka $\mathrm{Y}$, Higuchi T, Amanuma M, Endo K: Detection of metastatic lesions from malignant pheochromocytoma and paraganglioma with diffusionweighted magnetic resonance imaging: comparison with 18FFDG positron emission tomography and 123I-MIBG scintigraph. Ann Nucl Med 2008, 22:395-401.

\section{doi:10.1186/1477-7819-10-82}

Cite this article as: Cossetti et al:: Whole body diffusion for metastatic disease assessment in neuroendocrine carcinomas: comparison with OctreoScan ${ }^{\circledR}$ in two cases. World Journal of Surgical Oncology 2012 10:82.

\section{Submit your next manuscript to BioMed Central and take full advantage of:}

- Convenient online submission

- Thorough peer review

- No space constraints or color figure charges

- Immediate publication on acceptance

- Inclusion in PubMed, CAS, Scopus and Google Scholar

- Research which is freely available for redistribution 